\title{
Diffraction tomography using multimode surface waves
}

\author{
T. Meier, ${ }^{1}$ S. Lebedev, G. Nolet, and F. A. Dahlen \\ Department of Geosciences, Princeton University, Princeton, New Jersey
}

\begin{abstract}
A new method is described that makes it feasible to include scattered and converted surface waves into waveform inversions for the three-dimensional (3-D) structure of the Earth. The single scattering (Born) approximation forms the basis of the method. In order to minimize the amplitude of the scattered wave field, the background model is first adapted to correct for nonconverted, forward-scattered wave energy. We then perform Born inversion of the difference between the measured and synthetic waveforms, including a suite of Love and Rayleigh modes. The Born approximation yields linear equations of the form $\mathbf{A} \delta \gamma=\delta \mathbf{u}^{\mathrm{Bom}}$, which allow the determination of the three-dimensional perturbations $\gamma$ to the background model from the scattered wave field $\delta \mathbf{u}^{\text {Born }}$. This procedure is followed separately for each source-receiver pair to allow for optimized background models for each signal, as well as to minimize the computational burden. We winnow the data vector for each path by performing singular value decomposition using a diagonalization of $\mathbf{A A}^{T}$. In a realistic example we found that each vertical component seismogram yields 30-40 linear constraints on the 3-D Earth, significantly more than with conventional pure-path (WKBJ) inversions. In a synthetic test, one seismogram is shown to be able to image a simple model of a point scatterer off the great circle. As a spin-off of the formulation of the multimode inverse scattering problem, we not only obtain a series of eigenvectors that rank the sensitivity of a seismogram to Earth structure in a series of geometrical patterns, we also can compute the surface wave equivalent of a Fresnel zone.
\end{abstract}

\section{Introduction}

Seismic surface waves yield information about the upper part of the Earth, in particular about the shear wave velocity $\boldsymbol{\beta}^{\text {true }}$. In the case of long-period multimode surface waves the sensitivity extends to the whole upper mantle. The potential horizontal resolution of the waves increases with the frequency. However, it is well known that high-frequency waves are prone to scattering and mode conversions. Where mode coupling theory can deal with this at normal mode frequencies, a more approximate approach is needed for regional surface waves.

Different methods for the regional inversion of multipathed surface waves have been developed. Snieder [1986a] and Snieder and Nolet [1987] developed the Born approximation for surface waves, and Snieder $[1988 \mathrm{a}, \mathrm{b}]$ described a method for the joint inversion of many waveforms. Snieder included Love/Rayleigh fundamental mode conversions, but the limitations of com-

\footnotetext{
${ }^{1}$ Now at Friedrich Schiller University Jena, Jena, Germany.
}

Copyright 1997 by the American Geophysical Union.

Paper number 96JB03227.

0148-0227/97/96JB-03227\$09.00 puter memory precluded a full multimode treatment of scattering.

It is important to distinguish between inversions of the in situ shear velocity and the mapping of local phase velocities. Yomogida and $A k i$ [1987] also use the Born approximation but ignore mode conversions and model the wave propagation by assuming the wave propagates with a local (i.e., structural) phase velocity, for which they invert phase and amplitude data. Tanimoto [1990] avoids a simple Born approach on the local phase velocity but still inverts for the latter. In this paper we are concerned with the direct inversion of shear velocity (and possibly density). Wielandt [1993] pointed out that a difference exists between the dynamical (i.e., measured) and the structural phase velocity for nonplane waves. Friederich et al. [1994] and Friederich and Wielandt [1995] proposed a method for the determination of the amplitude and phase of the wave field of the fundamental Rayleigh mode using a regional network and inverted for the structural phase velocity beneath the network to derive a three-dimensional Earth model.

A coupled mode approach for the modeling of surface waves was developed by Kennett [1984], Odom [1986], Maupin [1988], and Bostock [1992]. Based on this approach, Marquering and Snieder [1995] developed an approximate inversion method which takes multiple forward scattering and mode coupling into account. 
All of the above methods lead to very heavy, often unmanageable computations if mode conversions are included and if all data have to be inverted jointly. In order to allow for inversion of many data, we shall in this paper develop a strategy that combines the Born approach with a partitioning of the inversion.

Nolet [1990] has shown how the partitioning of waveform inversions (PWI) into a nonlinear and linear step may greatly facilitate the interpretation of large data sets. In this paper we not only use PWI for computational efficiency but also to keep errors in the Born approximation under control. This method consists of two major steps. A first nonlinear waveform inversion is carried out for each path separately. Such a nonlinear inversion is possible because only a small set of parameters is able to describe an average model for the path. The second (linear) step combines the one-dimensional models for different paths to obtain a three-dimensional model. The method was successfully used for imaging the shear wave velocity in the upper mantle beneath Central Europe, the Tornquist Zone, and the southwestern part of the East European Platform [Zielhuis and Nolet, 1994]. Recently, PWI was applied to construct three-dimensional models for the upper mantle beneath North America [Van der Lee, 1996] and Philippine Sea [Lebedev et al., subm. to Geophys. Res. Lett., 1997].

The waveform modeling is based on the WKBJ approximation, inverting for a laterally homogeneous average model along each path. Synthetic waveforms are calculated by the summation of surface wave modes. The method is limited to a frequency range where the WKBJ approximation is valid. Smooth average models leave a discrepancy at higher frequencies in the seismogram, which we shall attempt to model in this paper with scattered modes using the Born approximation. The WKBJ step is essential to keep the discrepancy small, a major condition for the validity of Born, which is a single scattering approximation [Snieder, 1988a, b; Friederich et al., 1993].

\section{Partitioned Waveform Inversion}

In this section we briefly review the partitioned waveform inversion method and describe the determination of the background model for the Born inversion. We invert for only a single parameter, the $S$ wave velocity. We summarize our notation for the various models and the corresponding waveforms in Table 1.

Average perturbations $\overline{\delta \beta}$ to an initial one-dimensional model $\beta^{0}$ are determined by fitting a synthetic waveform $\mathbf{u}^{\text {WKBJ }}$ to an observed seismogram $\mathbf{u}^{\text {obs }}$. The seismogram may consist of only the vertical component or more components. The synthetic is calculated on the basis of the WKBJ approximation for a smooth laterally varying model and a first-order Taylor expansion of the wavenumber

$$
\mathbf{u}^{\mathrm{WKBJ}}=\sum_{\nu} \mathrm{A}_{\nu}^{0} \exp \left[i\left(k_{\nu}^{0}+\int_{0}^{a} \frac{\partial k_{\nu}^{0}}{\partial \beta} \overline{\delta \beta} d r\right) a \Delta\right]
$$

or

$$
\begin{aligned}
\mathbf{u}^{\mathrm{WKBJ}} & =\sum_{\nu} \mathbf{A}_{\nu}^{0} \exp \left[i\left(k_{\nu}^{0}+\overline{\delta k}_{\nu}\right) a \Delta\right] \\
& =\sum_{\nu} \mathbf{A}_{\nu}^{0} \exp \left[i \bar{k}_{\nu} a \Delta\right]
\end{aligned}
$$

where the sum extends over the modes, and $A_{\nu}^{0}$ is the amplitude vector of the mode for the initial model $\beta^{0}$. The wavenumber $k_{\nu}^{0}$ of each mode is perturbed by $\overline{\delta k}_{\nu}$ to fit the seismogram. The Fréchet kernels $\partial k_{\nu}^{0} / \partial \beta$ relate the wavenumber perturbation $\overline{\delta k_{\nu}}$ to the model perturbation $\overline{\delta \beta}$. The radius of the Earth is denoted by $a$ and the source-receiver angular distance by $\Delta$. The synthetic $\mathbf{u}^{\text {WKBJ }}$ is fitted to the data $\mathbf{u}^{\text {obs }}$ by minimizing a least squares penalty function

$$
F(\overline{\delta \bar{\beta}})=\int w(t)\left|\mathbf{u}^{\text {obs }}(t)-\mathbf{u}^{\mathrm{WKBJ}}(t, \overline{\delta \beta})\right|^{2} d t,
$$

where $w(t)$ is a weighting function designed to equalize the influence of different arrivals in the seismogram. The perturbation $\overline{\delta \beta}$ is assumed to be an average perturbation along the path:

$$
\overline{\delta \beta}=\frac{1}{\Delta} \int_{\text {path }}\left(\beta^{\text {true }}-\beta^{0}\right) d \Delta .
$$

Adding this to the initial model, we obtain a best fitting average one-dimensional (1-D) model $\bar{\beta}=\beta^{0}+\overline{\delta \beta}$ in each source-receiver great circle plane.

Equation (4) constitutes a linear constraint on the true three-dimensional (3-D) Earth $\beta^{\text {true }}$, where $\overline{\delta \beta}$ still depends on $r$. With the introduction of a new background model $\beta^{\text {ref }}$, which is the same for all paths, (4) is transformed into a system of linear equations of the form

$$
\int G_{i}^{\mathrm{WKBJ}} \delta\left(\beta^{\text {true }}-\beta^{\text {ref }}\right) d^{3} \mathbf{r}=q_{i}
$$

with uncorrelated errors in the transformed "data" $q_{i}$. The kernel $G_{i}^{\mathrm{WKBJ}}(\mathbf{r})$ samples the path. For details, see

Table 1. Symbols Used to Denote Various Models and Associated Waveforms

\begin{tabular}{llccl}
\hline & Model & Dimension & $\begin{array}{c}\text { Path } \\
\text { Dependent }\end{array}$ & Waveform \\
\hline Real Earth & $\beta^{\text {true }}$ & & & $\mathbf{u}^{\text {obo }}$ \\
Initial model & $\beta^{0}$ & $1-D$ & yes & $\mathbf{u}^{\mathbf{0}}$ \\
Average model & $\bar{\beta}=\beta^{0}+\overline{\delta \beta}$ & $1-D$ & yes & $\mathbf{u}^{\text {WKBJ }}$ \\
Perturbation of average model & $\delta \beta=\beta^{\text {true }}-\bar{\beta}$ & $3-D$ & yes & $\delta \mathbf{u}^{\text {Borm }}$ \\
Background model for inversion & $\beta^{\text {ref }}$ & $1-D$ & no & \\
Perturbation of $\beta^{\text {ref }}$ & $\delta \beta^{\text {ref }}=\beta^{\text {true }}-\beta^{\text {ref }}$ & 3-D & no & \\
\hline
\end{tabular}


Nolet [1990]. For many paths equations (5) are collected and inverted for perturbations $\delta \beta^{\text {ref }}=\beta^{\text {true }}-\beta^{\text {ref }}$. It is advantageous to allow the initial model $\beta^{0}$ for each path to differ from the reference model $\beta^{\text {ref }}$ to improve the accuracy of the WKBJ approximation.

\section{Born Approximation}

In most cases the residual

$$
\delta \mathbf{u}^{\text {Born }}=\mathbf{u}^{\text {obs }}-\mathbf{u}^{\text {WKBJ }}
$$

is small for low-passed signals but differs appreciably from zero if higher frequencies are included. In these cases $\delta u^{\text {Born }}$ can be seen as a perturbation to the waveform $u^{\text {WKBJ }}$ for the laterally homogeneous path-average model $\bar{\beta}=\beta^{0}+\overline{\delta \beta}$. The Born approximation relates the residual $\delta u^{\text {Born }}$ linearly to perturbations in the structural parameters $\delta \beta=\beta^{\text {true }}-\bar{\beta}$.

Snieder and Nolet [1987] formulate the Born approximation for a spherical Earth as follows:

$$
\begin{aligned}
\delta \mathbf{u}^{\text {Born }} & =\iint \sum_{\nu, \sigma} \mathbf{p}^{\nu} \frac{\exp i\left(\bar{k}_{\nu} a \Delta_{2}+\pi / 4\right)}{\left(\sin \Delta_{2}\right)^{1 / 2}} S^{\nu \sigma} \\
. & \frac{\exp i\left(\bar{k}_{\sigma} a \Delta_{1}+\pi / 4\right)}{\left(\sin \Delta_{1}\right)^{1 / 2}}\left(\mathbf{E}^{\sigma}: \mathbf{M}\right) \sin \theta d \theta d \phi .
\end{aligned}
$$

The indices $\sigma$ and $\nu$ denote excited and scattered modes, respectively. Both Rayleigh and Love modes are included in $\sigma$ and $\nu$. The vector $\mathbf{p}^{\nu}$ denotes the polarization vector of mode $\nu$ at the receiver, $\Delta_{1}$ is the distance between source and the scatterer at colatitude $\theta$ and longitude $\phi$, and $\Delta_{2}$ is the distance between scatterer and receiver. The moment tensor is denoted by $\mathbf{M}$ and the WKBJ strain tensor of mode $\sigma$ by $\mathbf{E}^{\sigma}$. The wavenumbers for the background model $\bar{\beta}$ are denoted by $\bar{k}_{\sigma}$ or $\bar{k}_{\nu}$.

The scattering matrix can be written as

$$
S^{\nu \sigma}=\int_{0}^{a}\left(K_{\mu}^{\nu \sigma} \delta \mu+K_{\rho}^{\nu \sigma} \delta \rho+K_{\lambda}^{\nu \sigma} \delta \lambda\right) d r
$$

The integration is over radius and $\delta \rho, \delta \mu, \delta \lambda$ are perturbations to the background model $\bar{\rho}, \bar{\mu}, \bar{\lambda}$. Here $\rho$ denotes the density whereas $\mu, \lambda$ are the Lamé parameters for an isotropic medium. To replace $\delta \mu, \delta \lambda$ by $\delta \alpha$, $\delta \beta, \delta \rho$ we make use of the linearized equations

$$
\begin{gathered}
\delta \mu=\bar{\beta}^{2} \delta \rho+2 \bar{\rho} \bar{\beta} \delta \beta, \\
\delta \lambda=\left(\bar{\alpha}^{2}-2 \bar{\beta}^{2}\right) \delta \rho+2 \bar{\rho} \bar{\alpha} \delta \alpha-4 \bar{\rho} \bar{\beta} \delta \beta,
\end{gathered}
$$

which can be obtained from $\lambda=\rho\left(\alpha^{2}-2 \beta^{2}\right)$ and $\mu=$ $\rho \beta^{2}$.

Because we want to invert for $S$ wave velocity perturbations $\delta \beta$ only, perturbations in the density and the $P$ wave velocity are assumed to be proportional to the perturbations in the $S$ wave velocity:

$$
\delta \rho=c_{\rho}(\bar{\rho} / \bar{\beta}) \delta \beta,
$$

$$
\delta \alpha=c_{\alpha}(\bar{\alpha} / \bar{\beta}) \delta \beta
$$

This reduces the scattering matrix to

$$
S^{\nu \sigma}=\int_{0}^{a} K_{\beta}^{\nu \sigma} \delta \beta d r
$$

where

$$
\begin{aligned}
K_{\beta}^{\nu \sigma} & =K_{\mu}^{\nu \sigma}\left[\bar{\beta}^{2} c_{\rho}(\bar{\rho} / \bar{\beta}+2 \bar{\rho} \bar{\beta})\right]+K_{\rho}^{\nu \sigma} c_{\rho}(\bar{\rho} / \bar{\beta}) \\
& +K_{\lambda}^{\nu \sigma}\left[\left(\bar{\alpha}^{2}-2 \bar{\beta}^{2}\right) c_{\rho}(\bar{\rho} / \bar{\beta})+2 \overline{\rho \alpha} c_{\alpha}(\bar{\alpha} / \bar{\beta})-4 \bar{\rho} \bar{\beta}\right] .
\end{aligned}
$$

The residual $\delta u^{\text {Born }}$ becomes a linear function of the perturbation to the average model $\delta \boldsymbol{\beta}=\beta^{\text {true }}-\overline{\boldsymbol{\beta}}=$ $\beta^{\text {true }}-\beta^{0}-\overline{\delta \beta}$. Inserting $\delta \beta$ in (4) yields

$$
\overline{\delta \beta}=\frac{1}{\Delta} \int_{\text {path }}(\delta \beta+\overline{\delta \beta}) d \Delta
$$

and therefore

$$
0=\frac{1}{\Delta} \int_{\text {path }} \delta \beta d \Delta .
$$

Equation (16) implies that the average of $\delta \beta$ along the great circle path at any depth is zero. This is expected since $\bar{\beta}$ is an average model for the great circle plane.

For the purpose of inversion the perturbation $\delta \beta$ is expanded into three-dimensional basis functions

$$
\delta \beta(\mathbf{r})=\sum_{i} \gamma_{i} h_{i}(\mathbf{r})=\sum_{i} \gamma_{i} h_{i}^{\tau}(r) h_{i}^{\alpha}(\theta, \phi) .
$$

The basis functions $h_{i}$ are assumed to be orthogonal. In this paper we choose the angular basis functions $h_{i}^{a}$ equal to 1 for a small region $\theta_{1 i} \leq \theta \leq \theta_{2 i}, \phi_{1 i} \leq \phi \leq \phi_{2 i}$ and zero outside, and $h_{i}^{\tau}$ equal to 1 where $r_{1 i} \leq r \leq r_{2 i}$ and zero elsewhere. These regions are assumed to be small in comparison to the wavelength of the structural perturbations.

The scattering matrix is split up into contributions from the different basis functions

$$
S^{\nu \sigma}=\sum_{i} \gamma_{i}\left(\int_{0}^{a} K_{\beta}^{\nu \sigma} h_{i}^{\tau} d r\right) h_{i}^{a}=\sum_{i} \gamma_{i} S_{i}^{\nu \sigma} h_{i}^{a} .
$$

Equation (7) can then be rewritten as

$$
\begin{gathered}
\delta \mathbf{u}^{\text {Born }}=\sum_{i} \gamma_{i} \iint \sum_{\nu, \sigma} \mathbf{p}^{\nu} \frac{\exp i\left(\bar{k}_{\nu} a \Delta_{2}+\pi / 4\right)}{\left(\sin \Delta_{2}\right)^{1 / 2}} S_{i}^{\nu \sigma} \\
. \frac{\exp i\left(\bar{k}_{\sigma} a \Delta_{1}+\pi / 4\right)}{\left(\sin \Delta_{1}\right)^{1 / 2}}\left(\mathbf{E}^{\sigma}: \mathrm{M}\right) h_{i}^{a} \sin \theta d \theta d \phi .
\end{gathered}
$$

Replacing quantities such as $\mathbf{E}^{\sigma}$ by their values $\mathbf{E}_{i}^{\sigma}: \mathbf{M}$, $p_{i}^{\nu}, S_{i, \text { cent }}^{\nu \sigma}$, and $\left(\sin \Delta_{2 i}\right)^{1 / 2},\left(\sin \Delta_{1 i}\right)^{1 / 2}$ at the center of each cell we can simplify (19) to

$$
\begin{aligned}
\delta \mathbf{u}^{\text {Born }}= & \sum_{i} \gamma_{i} \sum_{\nu, \sigma} \mathbf{p}_{i}^{\nu} \frac{\exp i\left(\bar{k}_{\nu} a \Delta_{2 i}+\pi / 4\right)}{\left(\sin \Delta_{2 i}\right)^{1 / 2}} S_{i, \text { cent }}^{\nu \sigma} \\
& \cdot \frac{\exp i\left(\bar{k}_{\sigma} a \Delta_{1 i}+\pi / 4\right)}{\left(\sin \Delta_{1 i}\right)^{1 / 2}}\left(\mathrm{E}_{i}^{\sigma}: \mathrm{M}\right) s_{i}^{\nu \sigma} \cdot \quad(20)
\end{aligned}
$$


The quantity $s_{i}^{\nu \sigma}$ accounts for the possibly rapid fluctuations of the exponential functions in (19):

$$
s_{i}^{\nu \sigma}=\iint \exp i\left(\bar{k}_{\nu} a \delta \Delta_{2 i}+\bar{k}_{\sigma} a \delta \Delta_{1 i}\right) h_{i}^{a} \sin \theta d \theta d \phi
$$

where $\Delta_{1}=\Delta_{1 i}+\delta \Delta_{1 i}$ and $\Delta_{2}=\Delta_{2 i}+\delta \Delta_{2 i}$. For small wavenumbers $s_{i}^{\nu \sigma}$ will be approximately equal to the surface area $s_{i}$ where $h_{i}^{a}=1$.

The following abbreviations for (20) are introduced:

$$
\begin{aligned}
\delta \mathbf{u}^{\text {Born }} & =\sum_{i} \gamma_{i} \sum_{\nu, \sigma} \tilde{\mathbf{a}}_{i}^{\nu \sigma}\left(\mathbf{E}_{i}^{\sigma}: \mathbf{M}\right) \\
& =\sum_{i} \gamma_{i} \sum_{\nu, \sigma} \mathbf{a}_{i}^{\nu \sigma}=\sum_{i} \gamma_{i} \mathbf{a}_{i} .
\end{aligned}
$$

A real linear system of equations for $\gamma=\left(\gamma_{1}, \gamma_{2}, \ldots\right)$ can be obtained in the frequency domain:

$$
\begin{aligned}
& \Re\left[\delta \mathbf{u}^{\text {Born }}\left(\omega_{j}\right)\right]=\sum_{i} \gamma_{i} \Re\left[\mathbf{a}_{i}\left(\omega_{j}\right)\right], \\
& \Im\left[\delta \mathbf{u}^{\text {Born }}\left(\omega_{j}\right)\right]=\sum_{i} \gamma_{i} \Im\left[\mathbf{a}_{i}\left(\omega_{j}\right)\right],
\end{aligned}
$$

or in the time domain by a Fourier back transformation of (22):

$$
\delta \mathbf{u}^{\text {Born }}\left(t_{j}\right)=\sum_{i} \gamma_{i} a_{i}\left(t_{j}\right)
$$

In the time domain a time window is easier to apply. These linear systems of equations will be abbreviated by

$$
\delta \mathbf{u}^{\text {Born }}=\mathbf{A} \boldsymbol{\gamma} \text {. }
$$

Before linear equations like (25) for different paths are combined for an inversion, an approximation of $\mathbf{A}$ by using singular value decomposition (SVD) [Press et al., 1992] is introduced in order to reduce the number of equations. This method is akin to the method of ranking and winnowing proposed by Gilbert [1971]. SVD of A yields

$$
\mathbf{A}=\mathbf{U} \mathbf{\Lambda} \mathbf{V}^{T}=\tilde{\mathbf{U}} \tilde{\mathbf{\Lambda}} \tilde{\mathbf{V}}^{T}
$$

where $\Lambda$ is a diagonal matrix with the singular values of $\mathbf{A}$ on its diagonal. For one path the linear problem (25) is severely underdetermined. Therefore only a small number of the singular values are nonzero. A new diagonal matrix $\tilde{\boldsymbol{\Lambda}}$ excluding zero and small singular values is used to quell the indeterminacy. The columns of the corresponding matrices $\tilde{\mathbf{U}}, \tilde{\mathbf{V}}$ constitute a basis for the range of $\mathbf{A}$ and the particular solution, respectively. If the number of data is denoted by $m$, the number of unknowns by $n$, and the number of nonzero singular values by $m^{\prime}$, the matrices are of the following types U: $(m \times n), \Lambda:(n \times n), \mathrm{V}:(n \times n), \tilde{\mathrm{U}}:\left(m \times m^{\prime}\right)$, $\tilde{\Lambda}:\left(m^{\prime} \times m^{\prime}\right), \tilde{\mathbf{V}}:\left(n \times m^{\prime}\right)$. Using (26) and the orthogonality $\tilde{\mathbf{U}}^{T} \tilde{\mathbf{U}}=I$, the linear system (25) can be transformed to

$$
\tilde{\mathbf{V}}^{T} \gamma=\tilde{\mathbf{\Lambda}}^{-1} \tilde{\mathbf{U}}^{T} \delta \mathbf{u}^{\text {Born }}=\eta
$$

The number of equations is reduced from $m$ in (25) to $\boldsymbol{m}^{\prime}$ in (27). From (27) the covariance matrix $\mathbf{C}_{\eta}=$ $E\left[\eta_{i} \eta_{j}\right]$ can be obtained:

$$
\mathbf{C}_{\eta}=\tilde{\mathbf{\Lambda}}^{-1} \tilde{\mathbf{U}}^{\mathbf{T}} \mathbf{C}_{\boldsymbol{u}} \tilde{\mathbf{U}} \tilde{\mathbf{\Lambda}}^{-1}
$$

or with $\mathbf{C}_{u}=\sigma_{u}^{2} \mathbf{I}$,

$$
\mathbf{C}_{\eta}=\sigma_{u}^{2} \tilde{\Lambda}^{-2}
$$

Multiplying (27) by $\tilde{\Lambda} / \sigma_{u}$ corresponds to a normalization of (27) to unit variance.

If the number $n$ of unknown elements in $\gamma$ is large, SVD of $\mathbf{A}$ is very time and space consuming. Because for one path the number of data $m$ is smaller than the number of unknowns $n$, it is much more efficient to consider diagonalization of $\mathbf{A A}^{T}$, even though the numerical precision can decrease. With $\gamma=\mathbf{A}^{T} \mathbf{y},(25)$ can be written as

$$
\delta \mathbf{u}^{\text {Born }}=\mathbf{A A}^{T} \mathbf{y}
$$

Diagonalization of $\mathbf{A} \mathbf{A}^{\mathbf{T}}$ yields

$$
\begin{gathered}
A A^{T}=\mathbf{U} \Lambda^{2} \mathbf{U}^{T}=\tilde{\mathbf{U}} \tilde{\Lambda}^{2} \tilde{\mathbf{U}}^{T}, \\
\delta \mathbf{u}^{\text {Born }}=\tilde{\mathbf{U}} \tilde{\Lambda}^{2} \tilde{\mathbf{U}}^{T} \mathrm{y} .
\end{gathered}
$$

From (32) we find that

$$
\tilde{\Lambda}^{-1} \tilde{\mathbf{U}}^{T} \delta \mathbf{u}^{\text {Born }}=\tilde{\Lambda} \tilde{U}^{T} \mathbf{y}=\eta .
$$

Finally, using (26) we can calculate

$$
\tilde{\mathbf{V}}^{T}=\tilde{\mathbf{\Lambda}}^{-1} \tilde{\mathbf{U}}^{T} \mathbf{A} .
$$

Using (33) and (34) the linear system of (27) can be obtained by diagonalization of $\mathbf{A A}^{T}$.

\section{Example 1}

Figures 1 and 2 illustrate properties of the Born approximation using a source-receiver pair on the Philippine Sea Plate. Figure 1 shows the absolute value of a scattering matrix at $25 \mathrm{mHz}$ for mode conversions from Love to Rayleigh modes as well as between Rayleigh modes for a single scatterer; 15 Love and 15 Rayleigh modes are considered. The scatterer is located at $10^{\circ} \mathrm{N}$, $125^{\circ} \mathrm{E}$ and consists of $5 \%$ perturbations in $\bar{\alpha}$ and $\bar{\beta}$ and $2.5 \%$ in $\bar{\rho}$ between 7 and $410 \mathrm{~km}$ depth ( $c_{\rho}=0.5$ and $\left.c_{a}=1.0\right)$. The horizontal extent of the scatterer is 80 $\mathrm{km}$ by $80 \mathrm{~km}$. The plots on the left show the dimensionless elements of the matrix $S_{i, \text { cent }}^{\nu \sigma} s_{i}$, whereas the plots on the right show the amplitudes $\left|a_{i}^{\nu \sigma}\right|$ of the resulting perturbations to the vertical component waveform at the receiver.

The scattering matrix is shown for near-forward scattering with a $30^{\circ}$ change in the direction of propagation. The scattering matrix is dominated by scattering without mode conversion as well as by scattering between neighboring Rayleigh modes. The scattering matrix is symmetric for scattering between Rayleigh modes. Love modes are scattered mainly to Rayleigh modes with a mode number which is increased by 1 or 2 . The ninth 

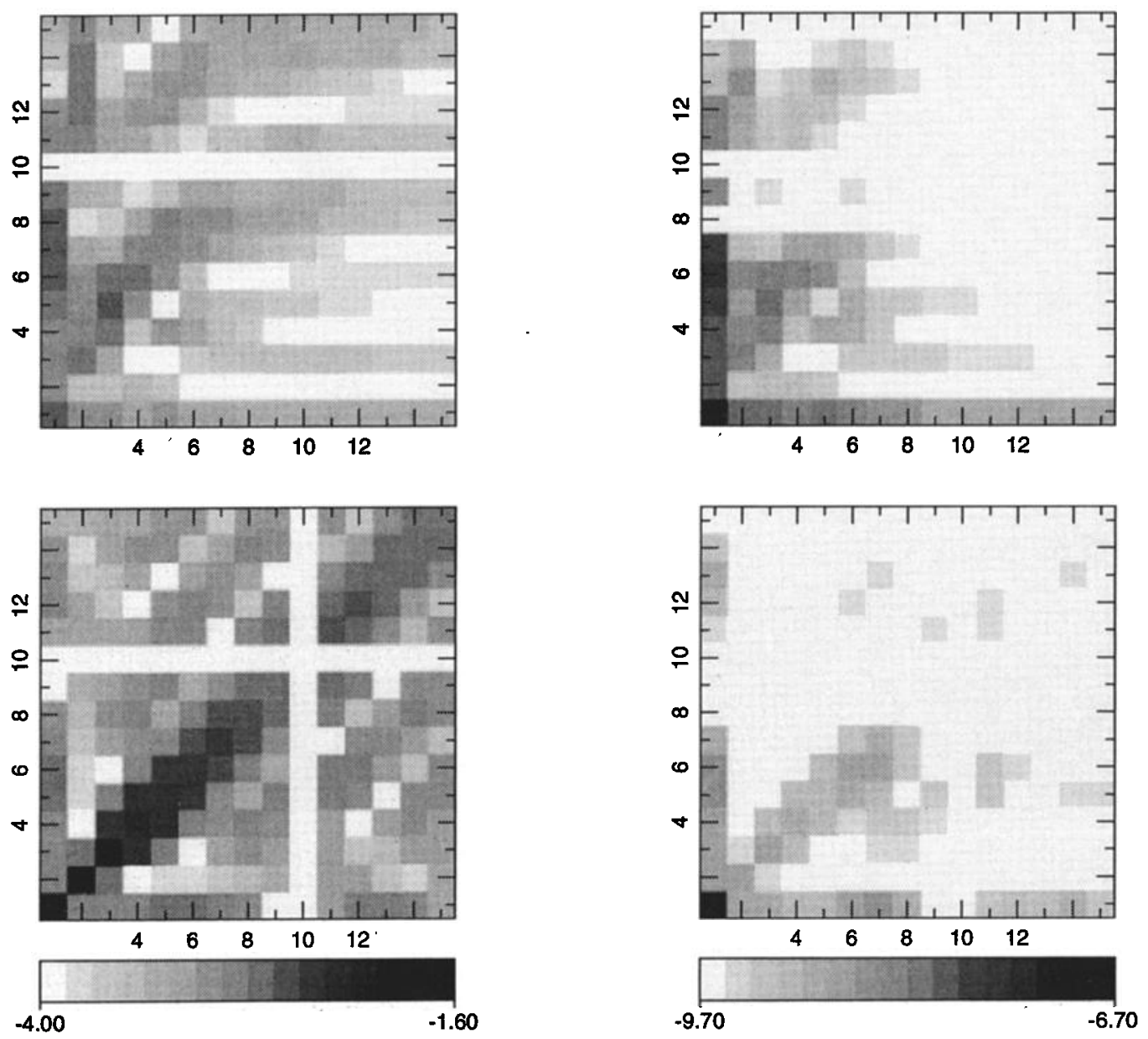

Figure 1. (left) Scattering matrix $S^{\nu \sigma}$ for a scatterer at $10^{\circ} \mathrm{N}, 125^{\circ} \mathrm{E}$ for a frequency of $25 \mathrm{mHz}$. (right) Amplitude $\left|a_{i}^{\nu \sigma}\right|$ of scattered waves on the vertical component in the Born approximation. (top) Love to Rayleigh conversion. (bottom) Scattering between Rayleigh modes. Absolute values are plotted using a base 10 logarithmic scale, that is, -4.00 means $10^{-4}$. The elements of the scattering matrix are dimensionless, whereas the amplitude of the scattered waves are given in meters. The scatterer has $5 \%$ perturbations in $\bar{\alpha}$ and $\bar{\beta}$ and $2.5 \%$ in $\bar{\rho}$ between 7 and $410 \mathrm{~km}$ depth. The horizontal extent of the scatterer is $80 . \mathrm{km}$ by $80 \mathrm{~km}$. Incoming modes are plotted on the $x$ axis, outgoing modes are plotted on the $y$ axis. See Figure 2 for the source-receiver configuration.

higher Rayleigh mode represents a Stoneley mode at the core-mantle boundary. The values of the eigenfunctions in the upper mantle for this mode are very small. For this mode the elements of the scattering matrix are also very small.

The amplitudes of the waveform perturbations or the scattered waves at the receiver are dependent on the scattering matrix and also on the excitation and the polarization vector for the receiver. The influences of both geometrical spreading and the size of the scatterer are the same for all mode conversions. For this scatterer in particular, Love to Rayleigh conversion is important because of the radiation pattern. The waveform perturbations are not symmetric for scattering between Rayleigh modes.

Figure 2 illustrates the Born approximation as a function of the lateral position of the scatterer (again at 25
$\mathrm{mHz}$. In the left column of plots we show scattering from the fundamental Rayleigh mode to itself, in the middle column the conversion from the first higher Rayleigh mode to the fundamental Rayleigh mode, and in the right column the conversion from the fundamental Love to the fundamental Rayleigh mode. The top two rows show the real parts of the waveform perturbations on the vertical component at the receiver as a function of the lateral position of the scatterer. The first row shows the perturbations without accounting for the effect of radiation pattern, and the second row includes the factor $\mathbf{E}_{i}^{\sigma}: \mathbf{M}$. In the notation of (22) the first row shows $\Re\left[\tilde{a}_{i}^{\nu \sigma}\right]$ and the second row shows $\Re\left[a_{i}^{\nu \sigma}\right]$ for three combinations of $\sigma$ and $\nu$. The index $i$ of the basis function varies. The depth dependence of the basis functions is the same for all lateral positions. The scatterers have $5 \%$ perturbations in $\bar{\alpha}$ and $\bar{\beta}$ and $2.5 \%$ in 

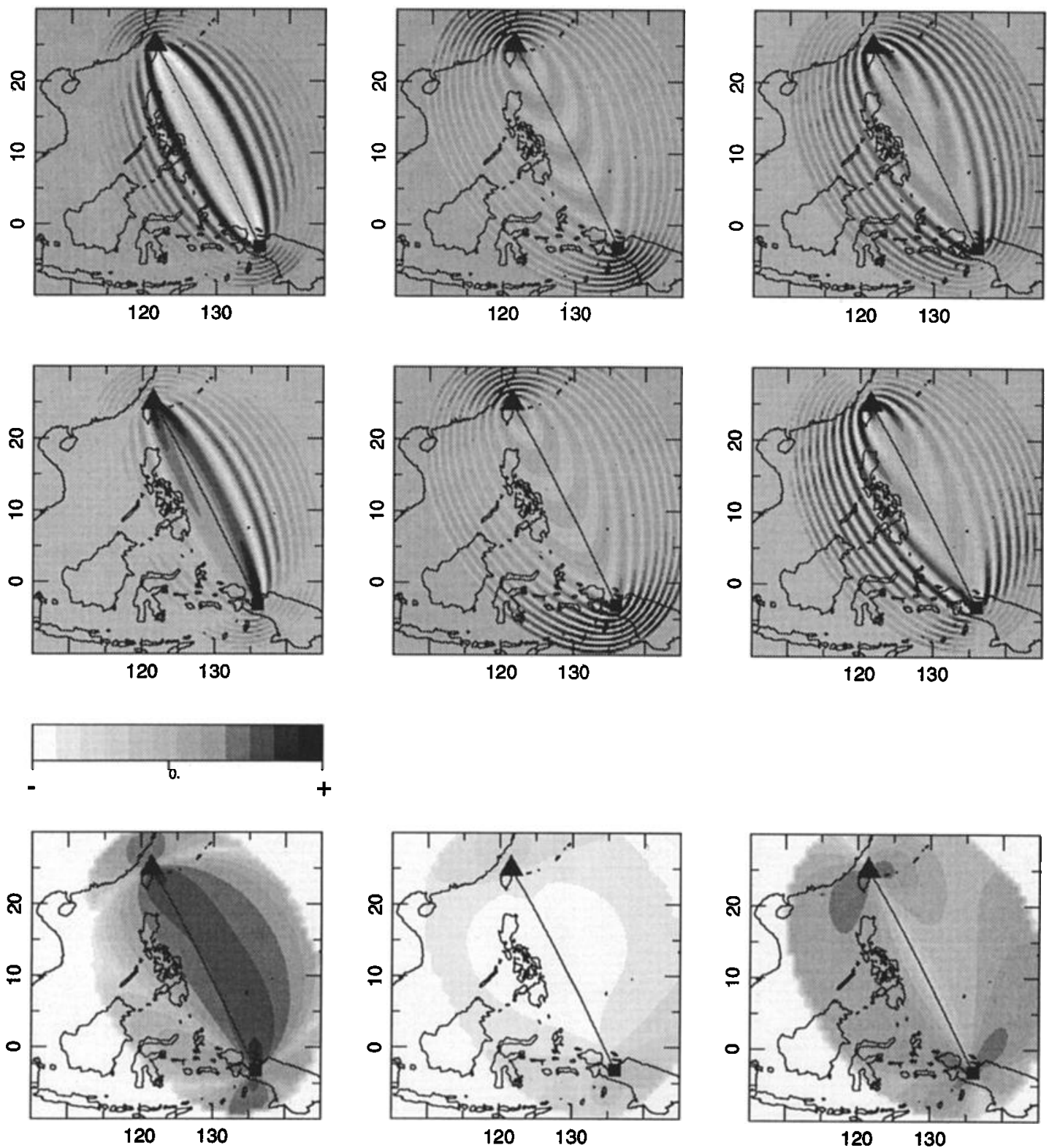

-9 .

$-6$.

Figure 2. Waveform perturbations as a function of the lateral position of the scatterer. Three examples of mode conversion are considered at a frequency of $25 \mathrm{mHz}$. (left) Scattering of fundamental Rayleigh to fundamental Rayleigh mode. (middle) Scattering of first higher Rayleigh to fundamental Rayleigh mode. (right) Scattering of fundamental Love to fundamental Rayleigh mode. (top) Real part of the waveform perturbations without radiation pattern accounted for $\left(\Re\left[\tilde{a}_{i}^{\sigma \nu}\right]\right)$. (middle) Real part of waveform perturbations, including effect of radiation pattern $\left(\Re\left[a_{i}^{\sigma \nu}\right\}\right)$. (bottom) Amplitude of the waveform perturbations ( $\left.\left|a_{i}^{\sigma \nu}\right|\right)$ in meters are given on a base 10 logarithmic scale. The scaling is the same for the three plots on the bottom. The plots in the first two rows are plotted independently. Exponents are shown in the legend, that is, -9 denotes $10^{-9}$. The scatterers are as in Figure 1. The source (square) is the event of April 13,1994 , at $3.1^{\circ} \mathrm{S}, 136.0^{\circ} \mathrm{N}$, depth $29 \mathrm{~km}$. The station (triangle) is the Global Seismic Network station TATO. The map shows a region around the Philippine Sea.

$\bar{\rho}$ between 7 and $410 \mathrm{~km}$ depth. In the top row curves of constant phase are visible. They have an elliptical shape if the wavenumber of the incoming and the scat- tered mode is equal or an "egg" shape otherwise. The amplitude along a curve of constant phase is modulated mainly by the angular dependence of the scattering and 
also by the geometrical spreading. For instance, in the case of the fundamental Rayleigh mode, forward and backward self-scattering have different signs. This explains the nodal plane visible between regions of forward and backward scattering. For fundamental Love to fundamental Rayleigh mode conversions the nodal plane coincides with the great circle plane. There is no such nodal plane for the conversion from the first higher Rayleigh to the fundamental Rayleigh mode; however, we see that backward scattering is stronger than forward scattering. In the second row the symmetry is lost because of the radiation pattern. In the third row amplitudes of the waveform perturbations $\left|a_{i}^{\sigma \nu}\right|$ are shown with the same scaling for the three plots. In this example, because of the radiation pattern, self-scattering from the fundamental Rayleigh mode is more sensitive to the structure northeast of the great circle. The radiation in the direction of the station is less than half of the maximum radiation, which is directed to the NNE. Scattering from the fundamental Love mode to the fundamental Rayleigh mode is stronger southwest of the great circle.

Upon linearizing the differentials $\delta \Delta_{1 i}$ and $\delta \Delta_{2 i}$ over a flat, square cell surface [Snieder, 1986b], the factor (21) can be approximated by the real number

$$
\begin{aligned}
s_{i}^{\nu \sigma}= & 4 \frac{\sin \left[a \Delta \theta\left(k_{\nu} \sin \varphi_{2 i}+k_{\sigma} \sin \varphi_{1 i}\right)\right]}{\left(k_{\nu} \sin \varphi_{2 i}+k_{\sigma} \sin \varphi_{1 i}\right)} \\
& \frac{\frac{\sin \left[a \Delta \theta\left(k_{\nu} \cos \varphi_{2 i}+k_{\sigma} \cos \varphi_{1 i}\right)\right]}{\left(k_{\nu} \cos \varphi_{2 i}+k_{\sigma} \cos \varphi_{1 i}\right)} .}{} .
\end{aligned}
$$

In (35) $\varphi_{2 i}$ is the azimuth toward the station, and $\varphi_{1 i}$ is the azimuth toward the source in the center of the cell, and $\Delta \theta$ denotes the angular half width of the cells. Figure 3 shows the ratio $s_{i}^{\nu \sigma} / s_{i}$, where $s_{i}$ is the angular cell surface, as a function of the position of the cell for $k_{\nu}=k_{\sigma}$. The source and receiver are assumed to be at $-20^{\circ} \mathrm{S}, 50^{\circ} \mathrm{E}$ and $20^{\circ} \mathrm{S}, 50^{\circ} \mathrm{E}$, respectively. In regions where the exponential functions in (21) are strongly fluctuating, the ratio $s_{i}^{\nu \sigma} / s_{i}$ becomes small. The ratio becomes larger for a smaller cell size and longer wavelengths. Equation (21) is dependent upon the shape of the cell. This is demonstrated in Figure 3d, where we have rotated the cells by $45^{\circ}$ with respect to those shown in Figure 3a.

Figures 4, 5, 6, and 7 demonstrate the decomposition of the matrix $\mathbf{A}$ governing the Born inversion. In Figure 4 (top) the waveform observed on the vertical component for the source-receiver configuration of Figure 2 is shown (solid line). It is compared with the synthetic waveform $u^{\text {WKBJ }}$ for the average model $\bar{\beta}$ resulting from the first step of the PWI (dashed line). The Harvard centroid moment tensor solution was used to compute the synthetic. The case shown is an extreme example, perhaps because the station is situated near a node in the radiation pattern of the fundamental Rayleigh mode; usually, the synthetic for an average model matches the waveform much better [Van der Lee, 1996, Lebedev et al., 1996]. In the example of Figure 4, neither the waveform within a window between the $S$
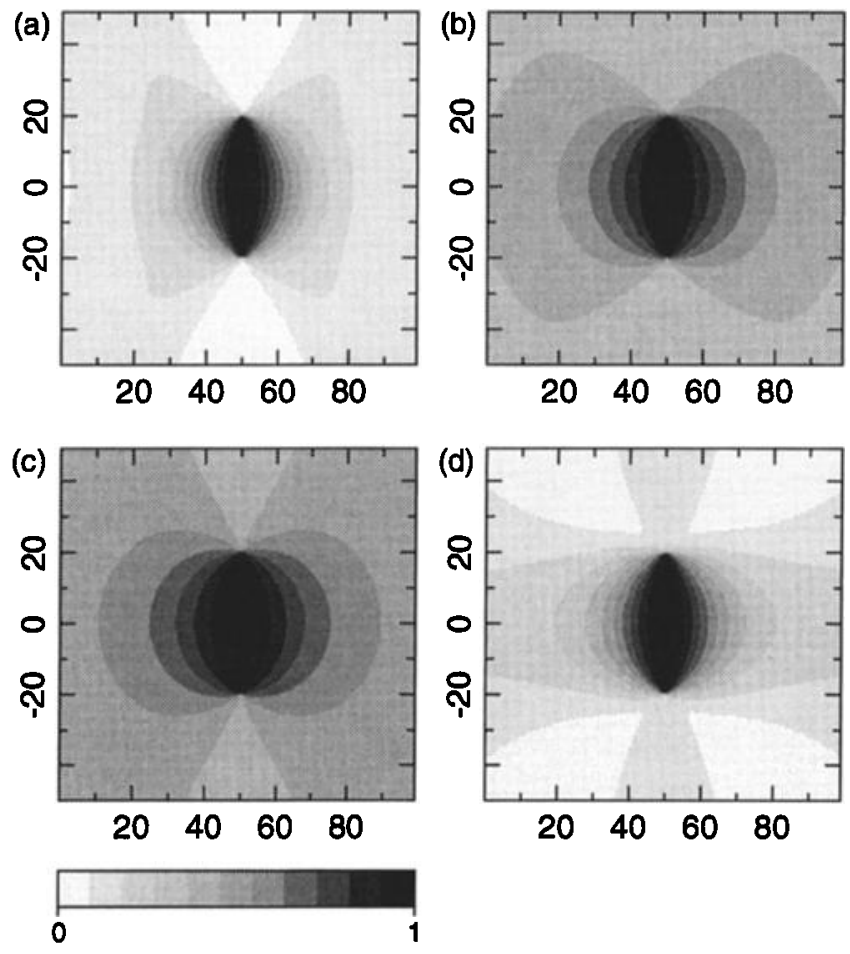

Figure 3. The ratio $s_{i}^{\nu \sigma} / s_{i}$ as a function of longitude and latitude. The source is at $-20^{\circ} \mathrm{S}, 50^{\circ} \mathrm{E}$ and the receiver at $20^{\circ} \mathrm{S}, 50^{\circ} \mathrm{E}$. Results are for $25 \mathrm{mHz}$. (a) $\Delta \theta=0.4^{\circ}$; phase velocity $4 \mathrm{~km} / \mathrm{s}$. (b) Like Figure $3 \mathrm{a}$, but phase velocity $6 \mathrm{~km} / \mathrm{s}$. (c) Like Figure $3 \mathrm{a}$, but $\Delta \theta=0.25^{\circ}$. (d) Like Figure 3a, but with cells rotated by $45^{\circ}$.

arrival and the fundamental mode (720 to $870 \mathrm{~s}$ ) nor the coda (after $950 \mathrm{~s}$ ) can be modeled with the WKBJ approximation. In the middle the residual $\delta u^{\text {Born }}$ between the observed waveform and the synthetic for the average model is shown (solid line). Figure 5 shows the first 47 singular values for the SVD of the matrix A. About $\mathbf{4 0}$ singular values are appreciably different from zero. The matrix $A$ has about 200 rows, 80 in this example a reduction of the number of rows by a factor of 5 is achieved. This is a desirable feature, since it reduces the storage requirements of the procedure considerably. A solution for this path can be computed using $\gamma=\tilde{V} \tilde{\Lambda}^{-1} \tilde{U}^{T} \delta u^{\text {Born }}$. The corresponding synthetic residual $\mathbf{A} \gamma$ is shown in Figure 4 (middle) with a dashed line. Figure 4 (bottom) shows the total synthetic waveform $\mathbf{u}^{\text {WKBJ }}+\mathbf{A} \gamma$ (dashed line) compared to the observed waveform $u^{\text {obe }}$ (solid line). The agreement is very good. We conclude that the Born approximation enables quite complicated waveforms to be modeled. Furthermore, the matrix $A$ can be approximated using about 40 nonzero singular values in this example.

For the construction of the basis functions $h_{i}$ in this example the depth range between 7 and $410 \mathrm{~km}$ was divided into four layers: 7 to $20 \mathrm{~km}, 20$ to $70 \mathrm{~km}, 70$ to $200 \mathrm{~km}$, and 200 to $410 \mathrm{~km}, 5 \%$ perturbations in $\bar{\alpha}$ and $\bar{\beta}$ and $2.5 \%$ in $\bar{\rho}$ are considered within these lay- 

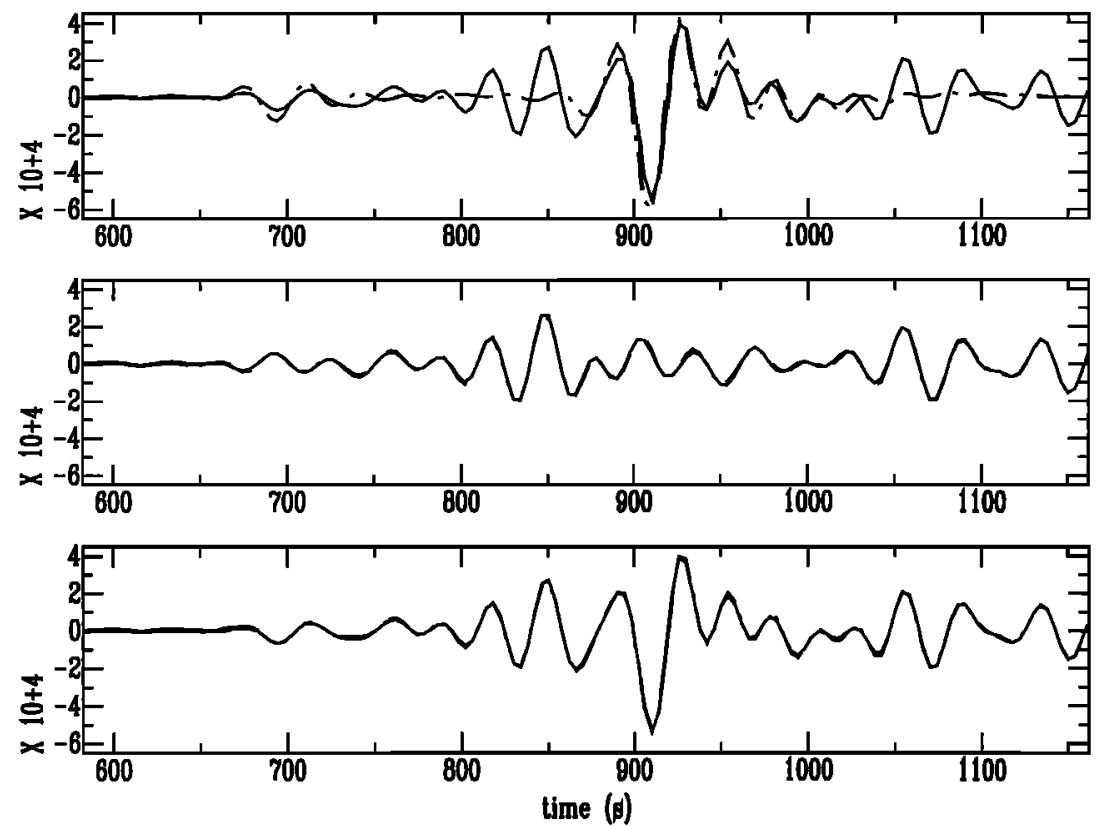

Figure 4. (top) Waveform for the source-receiver configuration in Figure 2 (solid line). Synthetic waveform $u^{\text {WKBJ }}$ for an average model (dashed line) resulting from the first step of the PWI. (middle) Residual $u^{\text {obs }}-\mathbf{u}^{\text {WKBJ }}$ (difference between data and synthetic for the average model, solid line) and synthetic residual $\delta \mathbf{u}^{\text {Born }}$ in the Born approximation (dashed line). (bottom) Comparison of observed waveform $\mathbf{u}^{\text {obs }}$ (solid line) and final synthetic $\mathbf{u}^{\text {WKBJ }}+\delta \mathbf{u}^{\text {Born }}$ (dashed line). Displacements are shown in nanometers in the frequency range 5 to $30 \mathrm{mHz}$. Read $X 10+4$ as $\times 10^{4}$.

ers for the construction of the basis function. In Figure 6 elements of the first, second, and fifteenth basis vector for the depth interval from 20 to $70 \mathrm{~km}$ are shown. Vectors corresponding to large singular values describe smooth model perturbations, whereas vectors corresponding to small singular values describe perturbations of short wavelength.

Since the condition number of $\mathbf{A} \mathbf{A}^{\boldsymbol{T}}$ is the square of the condition number of $A$, there is a valid concern for the numerical stability of our method. In Figure 7 we investigate the accuracy of the calculation of $\tilde{\mathbf{V}}$ using the diagonalization of $\mathbf{A} \mathbf{A}^{T}$. In Figure 7 (top) $\tilde{\mathbf{V}}^{T} \hat{\mathbf{V}}$ is shown for $\tilde{\mathbf{V}}$ as a result of SVD of $A$, and in Figure 7 (middle) $\tilde{\mathbf{V}}^{T} \tilde{\mathbf{V}}$ is shown for $\tilde{\mathbf{V}}$ as a result of diagonalization of $\mathbf{A A}^{T}$. Only columns of $\tilde{\mathbf{V}}$ corresponding to small singular values show deviations from the expected orthogonality if they are calculated using (34). In Figure 7 (bottom) dot products between columns of $\tilde{\mathbf{V}}$ calculated using SVD of $\mathbf{A}$ ( $x$ axes) and using diagonalization of $\mathbf{A A}^{\mathbf{T}}$ ( $y$ axes) are shown. In this example, diagonalization of $\mathbf{A} \mathbf{A}^{T}$ provides a very good estimate of $\tilde{V}$, especially for columns of $\tilde{\mathbf{V}}$ corresponding to the first 40 singular values.

\section{Inversion for Three-Dimensional Model}

Because of (17) the unknown coefficients and the basis functions are defined with respect to a background model $\bar{\beta}=\beta^{0}+\overline{\delta \beta}$ which is different for each path. We introduce a new background model $\beta^{\text {ref }}$ which is equal for all paths in order to obtain a system of linear equations governing the three-dimensional perturbations $\delta \beta^{\text {ref }}$. With (17) and

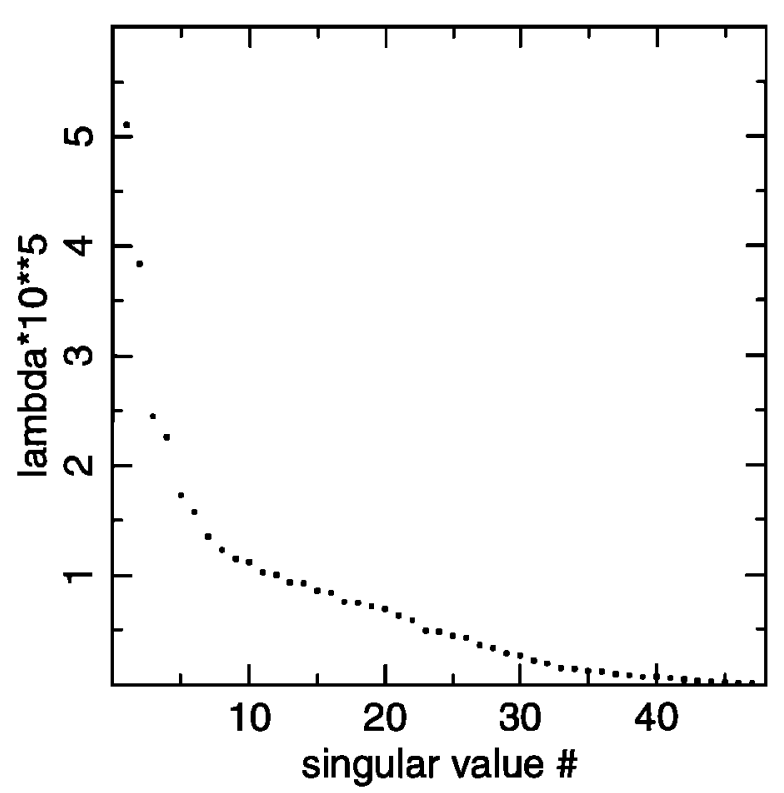

Figure 5. First 47 singular values of the matrix $\mathbf{A}$. 

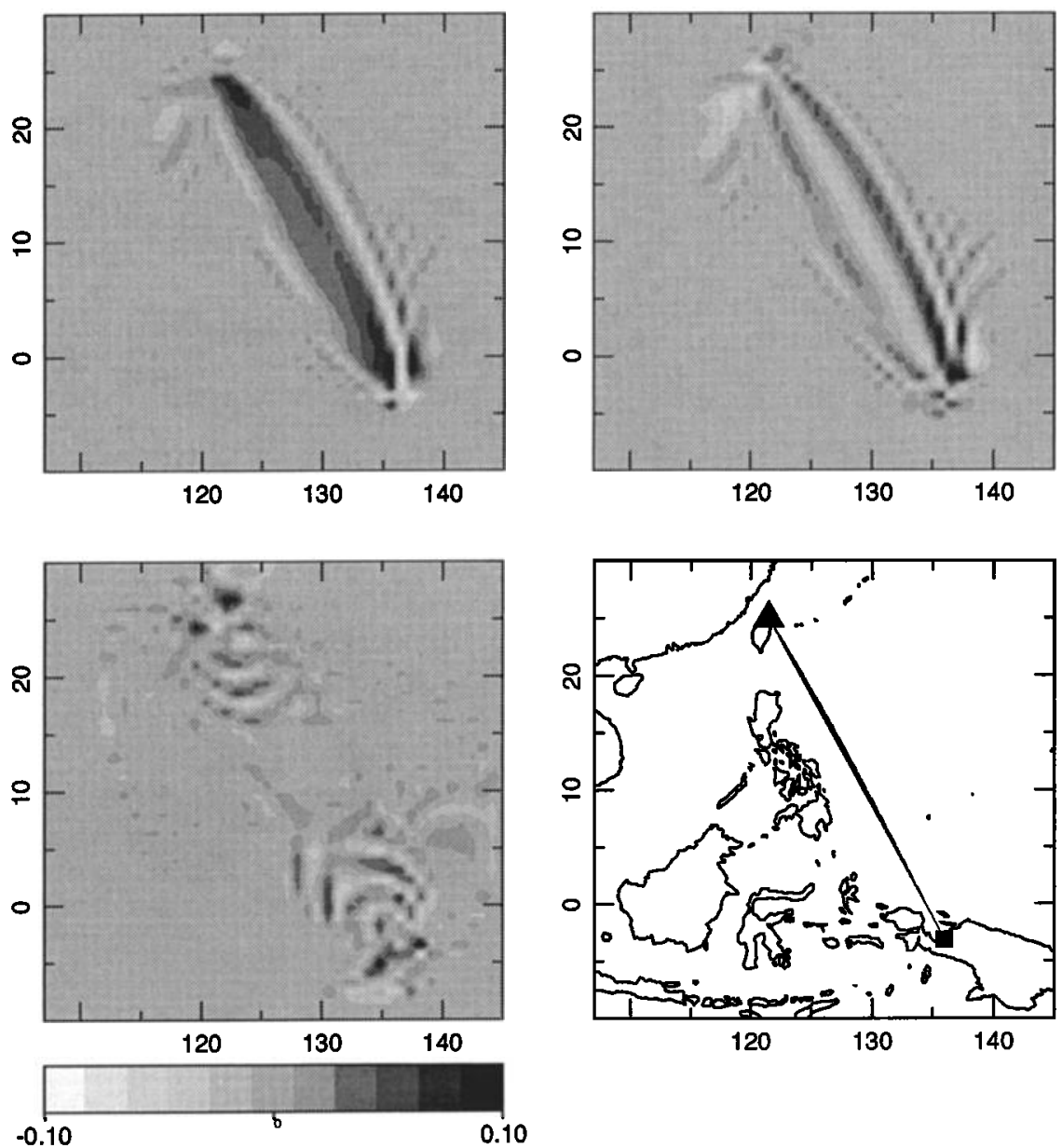

Figure 6. SVD of the matrix A yields a basis for the particular solution. Elements of the (top left) first, (top right) second, and (bottom left) fifteenth basis vectors for the depth range 20 to $70 \mathrm{~km}$. The source is denoted by a square; the receiver is denoted by a triangle.

$$
\int \tilde{h}_{j} h_{i} d^{3} \mathbf{r}=\delta_{j i}
$$

where $\tilde{h}_{j}=h_{j} / \int h_{j}^{2} d^{3} \mathbf{r}$, the coefficients $\gamma_{j}$ can be expressed by

$$
\int \tilde{h}_{j} \delta \beta d^{3} \mathbf{r}=\gamma_{j} .
$$

Using (37), (27) is written as

$$
\sum_{j} \tilde{V}_{j i} \int \tilde{h}_{j} \delta \beta d^{3} \mathbf{r}=\eta_{i}
$$

or in the general form

$$
\int G_{i} \delta \beta d^{3} \mathbf{r}=\eta_{i}
$$

A new background model $\beta^{\text {ref }}$ is introduced by

$$
\int G_{i}\left(\beta^{\text {true }}-\beta^{0}-\overline{\delta \beta}+\beta^{\text {ref }}-\beta^{\text {ref }}\right) d^{3} \mathbf{r}=\eta_{i},
$$

which yields

$$
\int G_{i} \delta \beta^{\text {ref }} d^{3} \mathbf{r}=\eta_{i}+\int G_{i}\left(\beta^{0}+\overline{\delta \beta}-\beta^{\text {ref }}\right) d^{3} \mathbf{r}=q_{i} .
$$

These equations are of the same form as the linear equations of the second step of the PWI (equation (5)). They represent linear constraints on the three-dimensional model of perturbations in the $S$ wave velocity. The right-hand side of (41) consists of two terms. The first term $\eta_{i}$ is due to perturbations of the laterally homogeneous average model $\bar{\beta}$ and the presence of a nonzero $\delta u^{B o r n}$. The second term depends on the difference between $\bar{\beta}$ and the background model of the inversion and on the result of the first step of the PWI.

For the final three-dimensional inversion, $\delta \beta^{\text {ref }}$ is expanded into basis functions

$$
\delta \beta^{\text {ref }}=\sum_{j} x_{j} f_{j} .
$$

For instance, the three-dimensional basis functions $f_{j}$ can be obtained by means of trilinear interpolation in a cubic grid containing the Earth [Van der Lee, 1996]. The linear system of equations for the determination of 

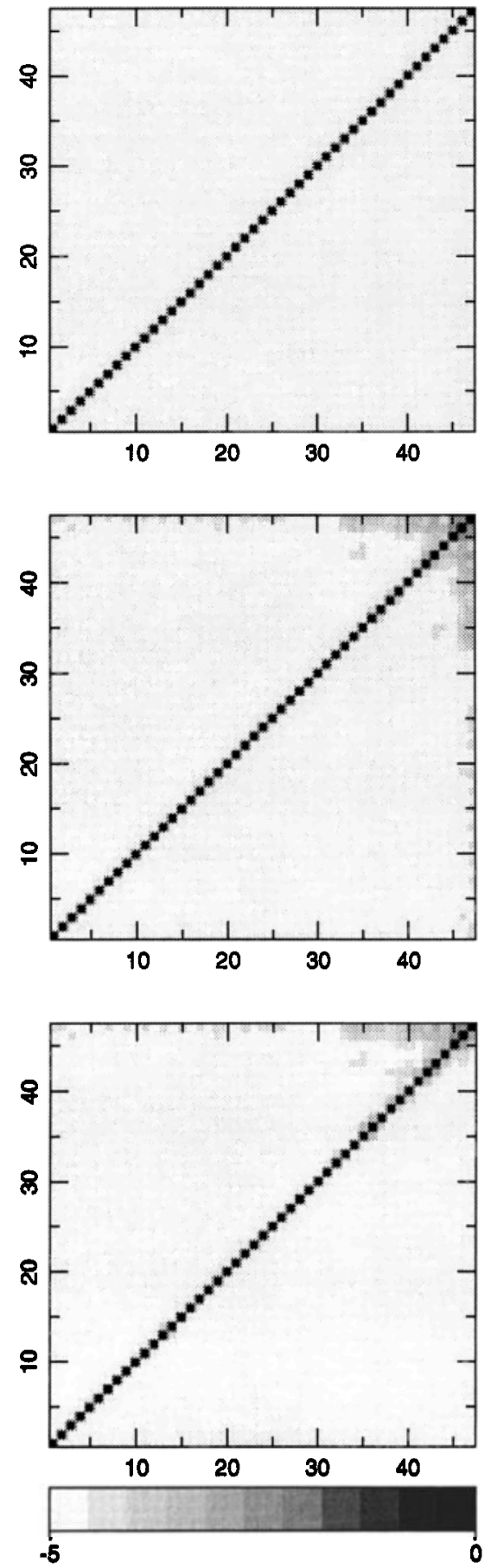

Figure 7. (top) $\tilde{\mathbf{V}}^{T} \tilde{\mathbf{V}}$ for $\tilde{\mathbf{V}}$ calculated using SVD of A. (middle) $\tilde{\mathbf{V}}^{T} \tilde{\mathbf{V}}$ for $\tilde{\mathbf{V}}$ calculated using diagonalization of $\mathbf{A A}^{T}$. (bottom) Dot product between columns of $\tilde{\mathbf{V}}$ calculated using SVD of $A$ ( $x$ axes) and diagonalization of $\mathbf{A A}^{T}$ ( $y$ axes). Base 10 logarithmic scaling is used.

the coefficients $x_{j}$ which has finally to be solved is then

$$
\sum_{j} x_{j} \int G_{i} f_{j} d^{3} \mathbf{r}=q_{i}
$$

For different paths, equations (43) are collected and simultaneously inverted by using LSQR [Paige and Saunders, 1982]. The large amount of disk space occupied by the matrix $\int G_{i} f_{j} d^{3} \mathbf{r}$ and the computation time is a matter of concern, but current technology already enables us to invert several hundred paths using a 2 Gbyte disk partition.

\section{Example 2}

The Born inversion is illustrated with a synthetic test. A synthetic residual for a single point scatterer is inverted. Figures 8 and 9 show a map view and cross sections of the resulting model for the Born perturbation $\delta \beta^{\text {ret }}$, for a scatterer located at $10^{\circ} \mathrm{N}, 125^{\circ} \mathrm{E}$, away from the great circle, and a scatterer on the great circle at $10^{\circ} \mathrm{N}, 130^{\circ} \mathrm{E}$, respectively. The synthetic residuals for the scatterers are shown in Figure 10. If only intramode scattering is considered, we would expect to see an ellipse through the position of the scatterer in a map view of the resulting model. Because mode conversions are taken into account, the maximum value in the inversion result reproduces the correct position of the scatterer fairly well. The maximum amplitude is too small, about $5 \%$ of the expected value, which is $200 \mathrm{~m} / \mathrm{s}$. It seems that the scatterer off the great circle is better reconstructed, probably because Love to Rayleigh mode conversions contribute to the synthetic waveform perturbation. The cross sections show that the vertical position of the scatterer between 20 and $70 \mathrm{~km}$ is almost reconstructed. These examples show the possibility of the lateral and vertical location of structural perturbations, and they address the problem of lateral and vertical resolution. It is expected that the inversion of waveforms for many different source-receiver configurations will increase the resolution.

We can also use the theory to study the "width" of a surface wave path. To this end we artificially set $\eta_{i}=0$ and $\beta^{\text {ref }}=\beta^{\circ}$ in (41) and solved the resulting equations for $\delta \beta^{\text {rof }}$. Figure 11 shows a map view of the solution at $100 \mathrm{~km}$ depth. Since this is a minimum norm solution, it is a measure of the spatial sensitivity of the seiomogram and a visualization of the surface wave equivalent of a Fresnel zone.

\section{Discussion and Conclusions}

The partitioning of the inverse problem for scattered waves is essential to make the method feasible because (1) it makes it possible to introduce different background models for different paths, thereby minimizing the scattered wave field $\delta u^{\text {Bora }}$, and (2) it reduces the inverse problem to the manageable size of a single data vector, rather than that of the 3-D model vector. The reduction in computational effort makes it possible for the first time to include conversions between a suite of higher Love and Rayleigh modes into the analysis.

Contrary to common practice, we do not invert for phase velocity maps as an intermediate step but solve directly for the 3-D Earth model. Phase velocity in- 

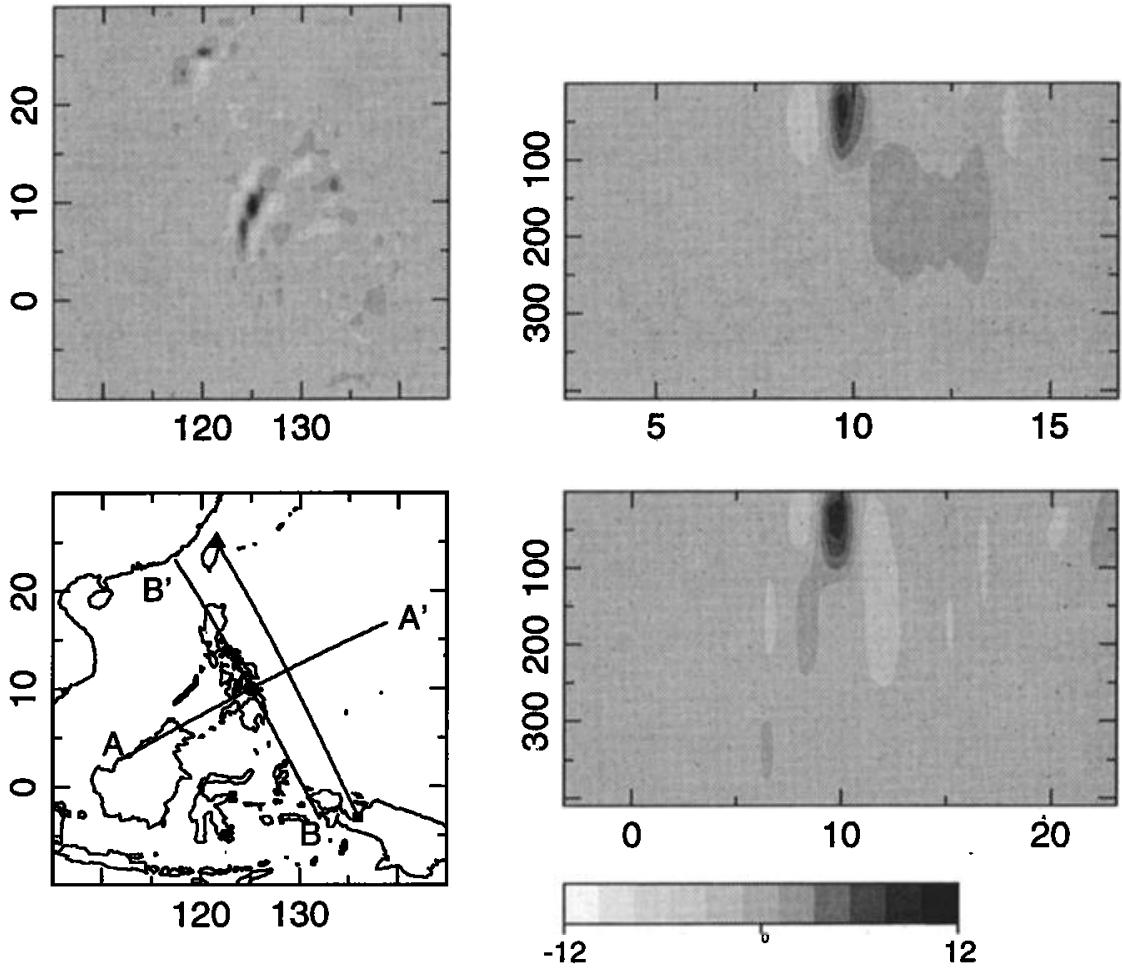

Figure 8. For one seismogram a synthetic inversion test is performed. The solid circle at $10^{\circ} \mathrm{N}$, $125^{\circ} \mathrm{E}$ denotes the position of the $80 \mathrm{~km} \times 80 \mathrm{~km}$ point scatterer. Its depth range is 20 to $70 \mathrm{~km}$. The source is denoted by a square; the receiver is denoted by a triangle. (top left) Map view of the resulting three-dimensional model at $50 \mathrm{~km}$ depth. (top right) Cross section of the resulting model, AA'. Perturbations $\delta \beta^{\text {ref }}$ are in meters per second. (bottom right) Cross section of the resulting model, $\mathrm{BB}^{\prime}$.
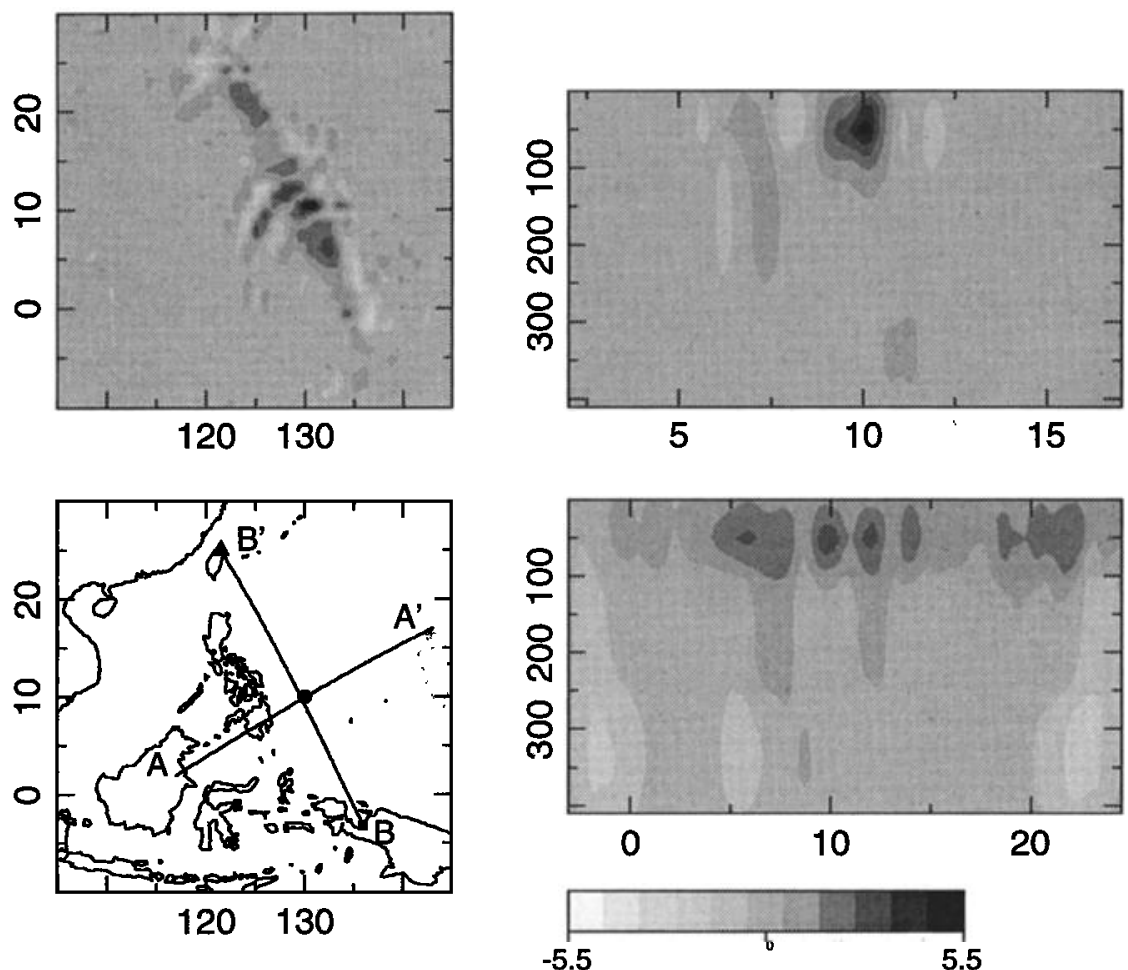

Figure 9. As in Figure 8, but now with the scatterer positioned at $10^{\circ} \mathrm{N}, 130^{\circ} \mathrm{E}$, on the great circle. 

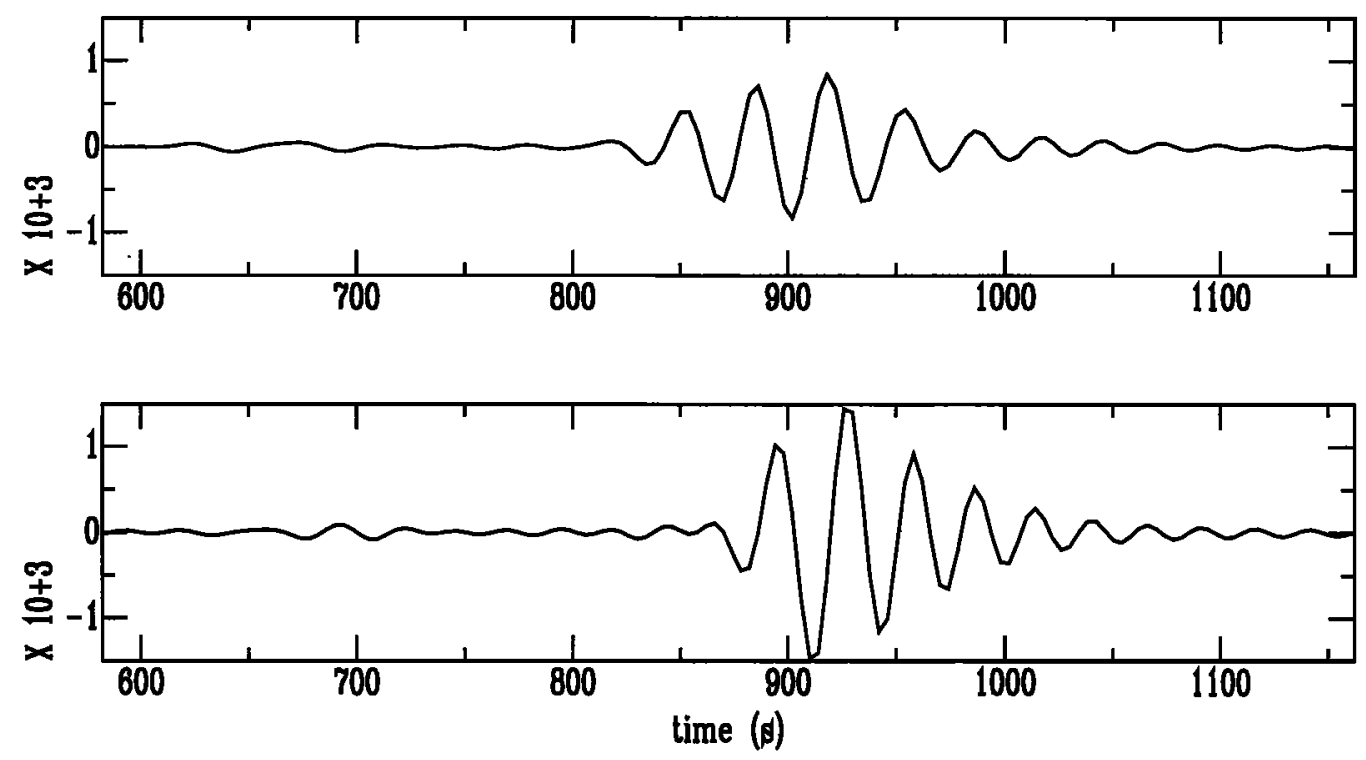

Figure 10. (top) Synthetic residual for scatterer in Figure 8. (bottom) Synthetic residual for scatterer in Figure 9. Read $X 10+3$ as $\times 10^{3}$.

version would be cumbersome in the case of multimode inversion; also, the fact that we allow for wave scattering violates the common assumption that structural and dynamic phase velocity are equal.

It is well known that the accuracy of the Born approximation decreases with increasing size of $\delta u^{\text {Born }}$, since multiple scattering is neglected. Because scattering increases with frequency, the accuracy of the method is a strong function of the low-pass filter applied to the seismograms, and theoretical errors can be kept under control. It is well known that the Born approximation

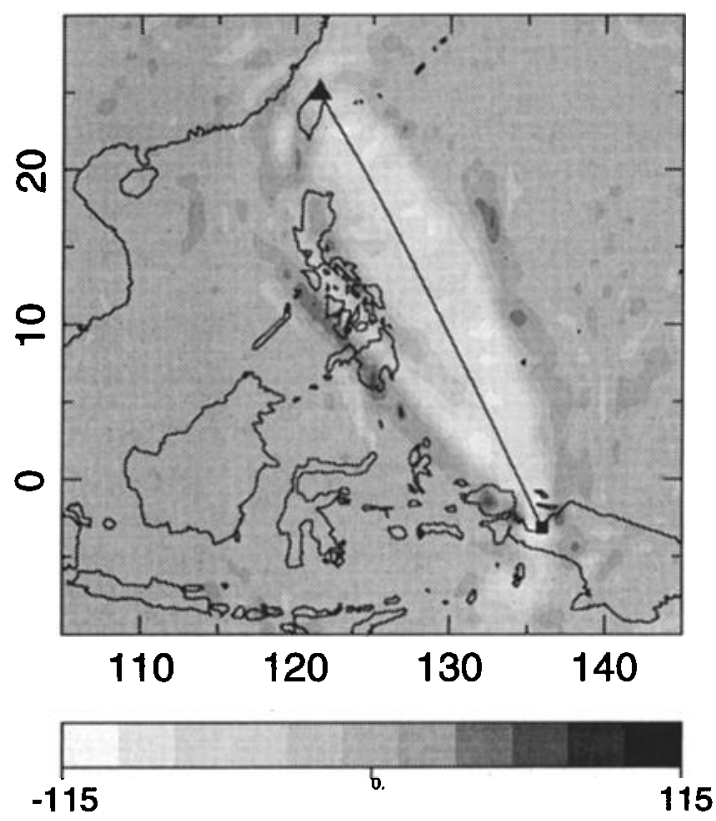

Figure 11. The input for this Born inversion is the average model for one path. A map view of the resulting model at $100 \mathrm{~km}$ depth is shown. Perturbations $\delta \beta^{\text {ref }}$ are in meters per second. is less accurate than WKBJ to model forward scattering; the reason is that the perturbed signal will only correctly modify the phase of the direct wave as long as the approximation $\exp (i \epsilon) \approx 1+i \epsilon$ is valid. However, for waves that scatter at a substantial angle, the direct wave plays no role and the Born approximation can be used with more confidence, as its widespread use in exploration seismics attests. Our application of WKBJ removes forward scattered energy that can be modeled by an average change of velocity along the path.

Note also that $\delta \mathbf{u}^{\text {Born }}$ need not be small with respect to the data $\mathbf{u}^{\text {obs }}$ for a particular path, but small with respect to the wave field in general. For example, the perturbed signal in Figure 4 is large with respect to the direct arrival of the Rayleigh wave, but in this case the station TATO is almost exactly on a node in the Rayleigh wave radiation pattern. A check against other records away from the node shows that the amplitude of the scattered wave is actually small. Such considerable influence of the radiation pattern is also evident from Figures 1 and 2 and explains an observation that has puzzled many seismologists: why seismograms of waves traveling along similar paths sometimes differ greatly in their content of scattered waves.

Our numerical experiments indicate that a single, vertical, seismogram low passed at $30 \mathrm{mHz}$ yields 30 40 independent constraints on Earth structure if allowance is made for off-path wave propagation. Within this subspace of eigenvectors the eigenvalues decrease only slightly more than 1 order of magnitude (Figure 5). This explains why we are able to obtain an almost perfect data fit without invoking excessive velocity gradients in the model. The question can be $r$ ised: can a similar fit still be obtained in a large inversion experiment with many paths that may locally overlap or be very close? Such an experiment is currently under way with real data. But even for, s:y, 100 paths the 
total number of independent constraints is still small compared to the number of model variables, which was 23,400 in our example, so that a good fit is still likely. Moreover, the introduction of multimode conversions in our procedure greatly enhances the accuracy of the inversions. If discrepancies remain, the two most likely causes are anisotropy (which can be handled within the same formalism as lateral heterogeneity) or errors in the source mechanisms.

The shape and orientation of the cell parameterization is not very critical near the great circle path but does have an influence at larger distance, as shown in Figure 3. Since the data constrain the Earth most strongly near the great circle (Figures 6 and 11), we expect little effect of the parameterization on the final outcome of the inversion. We conclude that we have developed an important new method which enables us to invert a large number of observed seismograms for 3-D Earth structure while taking the effects of multimode scattering into account to first order.

Acknowledgments. T.M. was supported by the German Academic Exchange Service under grant D/94/20517. Further support was provided by NSF through grants EAR9204386 and EAR-9218926.

\section{References}

Bostock, M.G., Reflection and transmission of surface waves in laterally varying media, Geophys. J. Int., 109, 411-436, 1992.

Friederich, W., and E. Wielandt, Interpretation of seismic surface waves in regional networks: Joint estimation of warefield geometry and local phase velocity: Method and numerical tests, Geophys. J. Int., 120, 731-744, 1995.

Friederich, W., E. Wielandt, and S. Stange, Multiple forward scattering of surface waves: Comparison with an exact solution and Born single-scattering methods, Geophys. J. Int., 112, 264-275, 1993.

Friederich, W., E. Wielandt, and S. Stange, Non-plane geometries of seismic surface wavefields and their implications for regional surface-wave tomography, Geophys. J. Int., 119, 931-948, 1994.

Gilbert, G.F., Ranking and winnowing gross Earth data for inversion and resolution, Geophys. J. R. Astron. Soc., 23, 125-128, 1971.

Kennett, B.L.N., Guided wave propagation in laterally varying media, I, Theoretical development, Geophys. J. $R$. Astron. Soc., 79, 235-255, 1984.

Lebedev, S., T. Meier, G. Nolet, and F.A. Dahlen, Probing the Westernmost Pacific upper mantle with surface waves, Eos Trans. $A G U, 7 \gamma(22)$, West. Pac. Geophys. Meet. Suppl., 85, 1996.

Marquering, H., and R. Snieder, Surface-wave mode coupling for efficient forward modelling and inversion of bodywave phases, Geophys. J. Int., 120, 186-208, 1995.
Maupin, V., Surface waves across 2-D structures: A method based on coupled local modes, Geophys. J., 93, 173-185, 1988.

Nolet, G., Partitioned waveform inversion and two-dimensional structure under the network of autonomously recording seismographs, J. Geophys. Res., 95, 8499-8512, 1990.

Odom, R.I., A coupled mode examination of irregular waveguides including the continuum spectrum, Geophys. J. $R$. Astron. Soc., 86, 425-453, 1986.

Paige, C.C., and M.A. Saunders, LSQR: An algorithm for sparse linear equations and sparse least squares, Assoc. Comput. Mach. Trans. Math. Software, 8, 43-71, 1982.

Press, W.H., S.A. Teukolsky, W.T. Vetterling, and B.P. Flannery, Numerical Recipes in FORTRAN, 2nd ed., Cambridge Univ. Press, New York, 1992.

Snieder, R., 3-D linearized scattering of surface waves and a formalism for surface wave holography, Geophys. J. $R$. Astron. Soc., 84, 581-605, 1986a.

Snieder, R., The influence of topography on the propagation and scattering of ourface waves, Phys. Earth Planet. Inter., 44, 226-241, $1986 \mathrm{~b}$.

Snieder, R., Large-scale waveform inversions of surface waves for lateral heterogeneity, 1 , Theory and numerical examples, J. Geophys. Res., 93, 12055-12065, $1988 \mathrm{a}$.

Snieder, R., Large-scale waveform inversions of surface waves for lateral heterogeneity, 2, Application to surface waves in Europe and the Mediterranean, J. Geophys. Res., 93, 12067-12080, 1988b.

Snieder, R., and G. Nolet, Linearized scattering of surface waves on a spherical Earth, J. Geophys., 61, 55-63, 1987.

Tanimoto, T., Modelling curved surface wave paths: Membrene surface wave synthetics, Geophys. J. Int., 102, 89$100,1990$.

Van der Lee, S., The Earth's upper mantle: Its structure beneath North America and the 660-km discontinuity beneath northern Europe, Ph.D. thesis, Princeton Univ., Princeton, N.J., 1996.

Wielandt, E., Propagation and structural interpretation of non-plane waves, Geophys. J. Int., 113, 45-53, 1993.

Yomogida, K., and K. Aki, Amplitude and phase data inversions for phase velocity anomalies in the Pacific Ocean basin, Geophys. J. R. Astron. Soc., 88, 161-204, 1987.

Zielhuis, A., and G. Nolet, Shear wave velocity variations in the upper mantle beneath central Europe, Geophys. $J$. Int., 117, 695-715, 1994.

F. A. Dahlen, S. Lebedev, and G. Nolet, Department of Geosciences, Guyot Hall, Princeton University, Princeton, NJ 08544. (e-mail: tony@weasel.princeton.edu; lebedev@geo.princeton.edu; guust@geo.princeton.edu)

T. Meier, Ricarda-Huch-Weg 11, D-07743 Jena, Germany. (e-mail: pet@rz.uni-jena.de)

(Received February 16, 1996; revised July 25, 1996; accepted October 15, 1996.) 